TITLE:

\title{
Hydrogen depth-profiling in chemical-vapor-deposited diamond films by high-resolution elastic recoil detection
}

\section{$\operatorname{AUTHOR}(S):$}

Kimura, K; Nakajima, K; Yamanaka, S; Hasegawa, M; Okushi, H

\section{CITATION:}

Kimura, K ... [et al]. Hydrogen depth-profiling in chemical-vapor-deposited diamond films by high-resolution elastic recoil detection. APPLIED PHYSICS LETTERS 2001, 78(12): 16791681

\section{ISSUE DATE:}

2001-03-19

URL:

http://hdl.handle.net/2433/39642

\section{RIGHT:}

Copyright 2001 American Institute of Physics. This article may be downloaded for personal use only. Any other use requires prior permission of the author and the American Institute of Physics. 


\title{
Hydrogen depth-profiling in chemical-vapor-deposited diamond films by high-resolution elastic recoil detection
}

\author{
Kenji Kimura ${ }^{\text {a) }}$ and Kaoru Nakajima \\ Department of Engineering Physics and Mechanics, Kyoto University, Kyoto 606-8501, Japan \\ Sadanori Yamanaka, Masataka Hasegawa, and Hideyo Okushi \\ Electrotechnical Laboratory, 1-1-4, Umezono, Tsukuba, Ibaraki 305-8568, Japan
}

(Received 14 November 2000; accepted for publication 27 January 2001)

\begin{abstract}
We have measured the hydrogen depth profiles in chemical-vapor-deposited diamond films by elastic recoil detection. Depth resolution of $\sim 0.23 \mathrm{~nm}$ is achieved using a high-resolution magnetic spectrometer. The hydrogen depth profile shows a sharp peak at surface, and the hydrogen coverage is estimated to be $1 \pm 0.3 \mathrm{ML}$, indicating formation of the monohydride structure. The surface peak has a small tail toward deeper region, which is ascribed to hydrogen atoms incorporated in a subsurface region. These subsurface hydrogen atoms might be the origin of the surface conductivity. (C) 2001 American Institute of Physics. [DOI: 10.1063/1.1356452]
\end{abstract}

Diamond is a promising material for electronic devices operating at high temperatures and/or high frequencies, as well as for high-power electronic devices. Recent investigation has shown that high-quality diamond films with atomically flat surfaces can be prepared by microwave-plasma chemical vapor deposition (CVD) under ultralow $\mathrm{CH}_{4} / \mathrm{H}_{2}$ conditions. ${ }^{1}$ The diamond film prepared by CVD has a $p$-type semiconducting layer at a surface region. ${ }^{2}$ The similar surface conductivity was also observed with natural diamond treated by hydrogen-rich plasma. ${ }^{3}$ This surface conductivity can be easily removed by oxidation with acid solutions or by annealing at $\sim 400{ }^{\circ} \mathrm{C},{ }^{2,4-7}$ suggesting that the surface conductivity is related to the hydrogen. Actually, secondary ion mass spectroscopy (SIMS) measurements revealed the existence of hydrogen in the surface region for both as-grown and hydrogenated samples but not for oxidized ones. ${ }^{7}$ The hydrogen-related luminescent states in the subsurface region were also observed by cathodoluminescence. ${ }^{8}$ Although the $p$-type semiconducting layer was utilized to fabricate electronic devices, ${ }^{9-11}$ the origin of the hydrogen induced conductive layer is not yet clarified.

There are several models for the hydrogen induced conductivity. One is the formation of shallow-accepter levels due to the hydrogen incorporated in the subsurface region of $\sim 20 \mathrm{~nm} .^{5-7,12,13}$ The model is strongly supported by the observed ideal rectification properties of Al-Schottky contacts to the CVD diamond, which indicates the existence of the depletion layer at the Al-diamond interface. ${ }^{13}$ Another model is the formation of negative charge states at the surface originating from the hydrogen termination, which induces surface accumulation of holes. ${ }^{14,15}$ Recently, Maier et al. have shown that exposure to air is essential for the hydrogen induced conductivity and proposed a model. ${ }^{16}$ A similar model was also proposed by Ri et al. ${ }^{17}$ In their model redox reaction in an adsorbed water layer provides the electron sink for the subsurface hole accumulation layer. Although the model

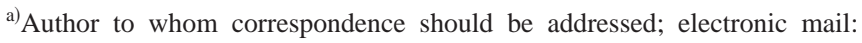
kimura@kues.kyoto-u.ac.jp
}

explains their findings, it is difficult to explain the observed $I-V$ characteristics of the Al-Schottky contacts with their model. $^{13}$

In spite of the hot debate between several groups claiming these models, important information, namely the hydrogen concentration in the subsurface region has been lacking. This is mainly because hydrogen is one of the most difficult elements for quantitative analysis. There are several measurements on the hydrogen depth profile by the elastic recoil detection (ERD $)^{18}$ and SIMS. ${ }^{7}$ The depth resolution of the conventional ERD (typically $\sim 10 \mathrm{~nm}$ ) is, however, not good enough for hydrogen profiling in the subsurface region. Although the depth resolution of SIMS is better, it gives only relative profiles. Moreover, the existence of a large number of surface hydrogen atoms, which is $1.6 \times 10^{15} \mathrm{~cm}^{-2}$ even for the monohydride structure, might cause the artificial subsurface hydrogen profile due to the ion mixing effects in SIMS. In the present letter, hydrogen profiles in the CVD diamond films are measured by high-resolution ERD with a depth resolution $\sim 0.2 \mathrm{~nm}$. The absolute hydrogen concentration in the subsurface region of the CVD diamond films is measured.

Undoped and B-doped diamond films were epitaxially grown on (001) surfaces of synthetic $\mathrm{Ib}$ diamond $(4 \times 4$ $\times 0.3 \mathrm{~mm}^{3}$ ) by microwave-plasma CVD. The details of the preparation are described elsewhere. ${ }^{7}$ The diamond films were mounted on a high-precision five-axis goniometer in a scattering chamber which was connected to a 4 MV Van de Graaff accelerator. A beam of $500 \mathrm{keV} \mathrm{C}^{+}$ions from the accelerator was collimated to $2 \mathrm{~mm}$ (vertical) $\times 1 \mathrm{~mm}$ (horizontal) and a divergence angle of less than $1 \mathrm{mrad}$ by a series of apertures. The beam current was monitored by a vibrating beam chopper and the typical beam current was $\sim 3 \mathrm{nA}$.

The diamond films were irradiated by the $\mathrm{C}^{+}$beam at an incident angle of $70^{\circ}$. The $\mathrm{H}^{+}$ions recoiled at $25^{\circ}$ with respect to the incident direction were energy analyzed by a $90^{\circ}$ sector magnetic spectrometer. The acceptance angle of the spectrometer was $0.2 \mathrm{msr}$. The spectrometer had an electrostatic quadrupole lens just before the magnet to correct the so-called kinematic broadening. The correction of the kine- 


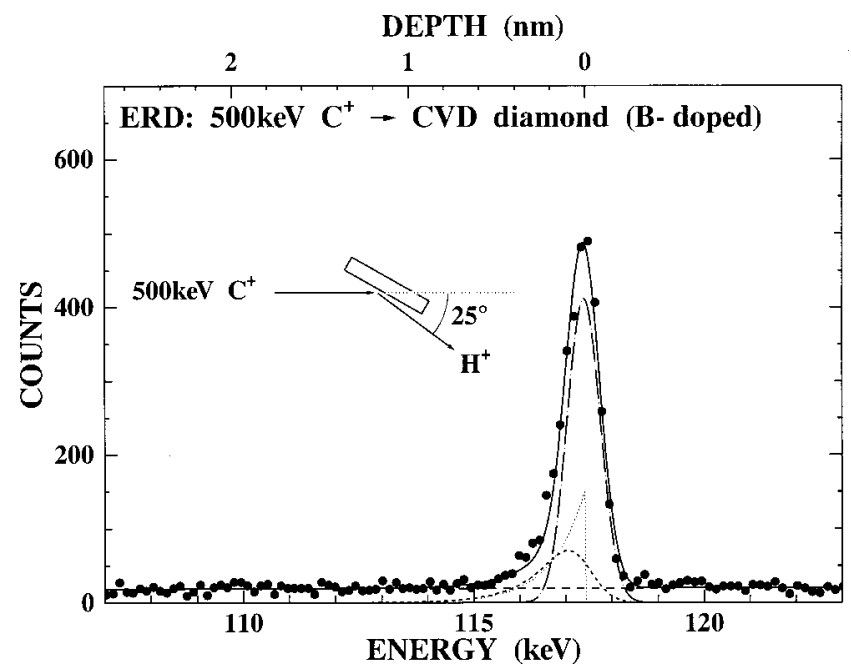

FIG. 1. Observed energy spectrum of recoiled $\mathrm{H}^{+}$for (001) surface of as-grown B-doped CVD diamond. The dose of the incident $500 \mathrm{keV} \mathrm{C}^{+}$ ions is about $3 \times 10^{15} \mathrm{~cm}^{-2}$. Estimated contributions due to the surface hydrogen (dot-dashed curve), the subsurface hydrogen (short-dashed curve), the background (long-dashed curve), and the sum of those (solid curve) are also shown.

matic broadening is essential to achieve a high depth resolution. ${ }^{19}$ The energy analyzed ions were detected by a one dimensional position sensitive detector (PSD) of 100 $\mathrm{mm}$ length which could move vertical direction. Not only the recoiled $\mathrm{H}^{+}$ions of energy $E$ but also scattered $\mathrm{C}^{+}$ions of $E / 12$ could pass through the spectrometer. These $\mathrm{C}^{+}$ions were rejected by means of an electrostatic deflector installed between the magnet and the PSD. ${ }^{20}$

Figure 1 shows the observed $\mathrm{H}^{+}$spectrum for a B-doped CVD diamond film. There is a peak at $\sim 117.4 \mathrm{keV}$, which corresponds to the hydrogen at the surface. The spectrum for the undoped CVD diamond film (not shown) is essentially the same as the presented B-doped spectrum. The energy scale was converted to depth scale using stopping powers estimated by TRIM code. ${ }^{21}$ The calculated depth scale is shown in the upper abscissa. The full width at half maximum of the surface hydrogen peak is about $0.9 \mathrm{keV}$ which corresponds to $0.23 \mathrm{~nm}$, showing that the depth resolution of the present system is better than $0.23 \mathrm{~nm}$ at the surface. Except for the peak the yield is fairly constant and almost the same level as the dark noise of the PSD. These background signals were fitted by a straight line as shown by a long-dashed line in Fig. 1.

Subtracting the background, the net hydrogen yield was derived. The yield was found to decrease with ion dose, indicating that hydrogen atoms are desorbed by the $\mathrm{C}^{+}$irradiation. The hydrogen coverage was estimated from the net yield using the PSD efficiency $(60 \%)^{22}$ and the $\mathrm{H}^{+}$fraction in the literature ${ }^{23}(80 \%$ at $117 \mathrm{keV})$. The obtained hydrogen coverage is $1.0 \pm 0.3 \mathrm{ML}\left(\mathrm{ML}=1.572 \times 10^{15} \mathrm{~cm}^{-2}\right)$ at zero dose. The error is mainly due to the uncertainty in the transmittance of the spectrometer. The present result indicates that the hydrogenated CVD diamond surface has a monohydride structure as was suggested by scanning tunneling microscopy observations ${ }^{14}$ and also suggested by a theoretical study. ${ }^{24}$

Looking at the shape of the hydrogen peak closely (Fig. 1 ), the peak has a small tail in the low energy side. This Downloaded 30 May 2007 to 130.54 .110.22. Redistribution subject

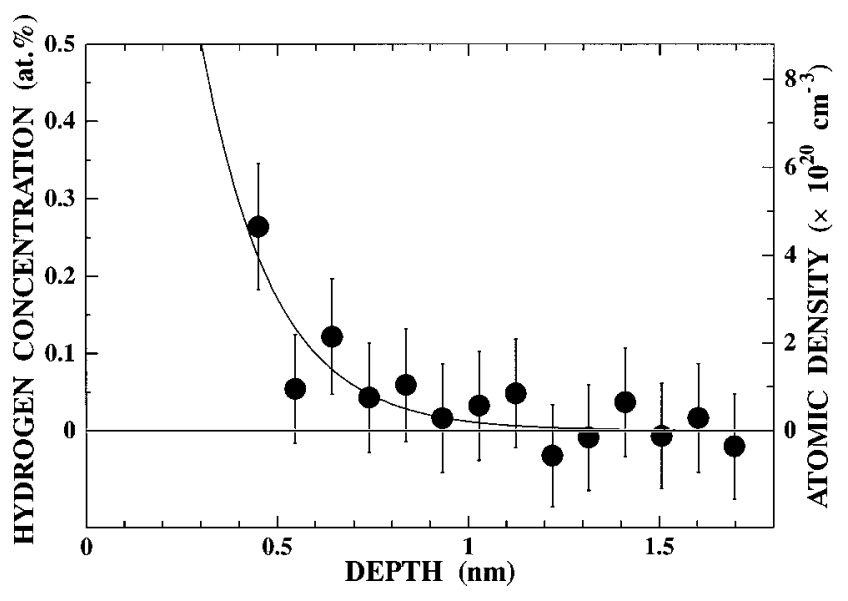

FIG. 2. Hydrogen concentrations derived from the tail of the surface peak (energy region of $E \leqslant 116 \mathrm{keV}$ in the energy spectrum shown in Fig. 1). Hydrogen concentration is less than the detection limit $(<0.05 \mathrm{at} / \%)$ at $d$ $\geqslant 1 \mathrm{~nm}$. The data is fitted by an exponential function as shown by a solid curve.

indicates that some hydrogen atoms are incorporated in a subsurface region. Assuming that the surface hydrogen peak is symmetric (see dot-dashed curve in Fig. 1), the signals at $E \leqslant 116 \mathrm{keV}$ can be ascribed to the subsurface hydrogen. The hydrogen depth profile derived from this energy region is shown in Fig. 2. The hydrogen concentration decreases very rapidly with increasing depth $d$ and is less than the detection limit of the present measurement $(\sim 0.05$ at. \% $=9 \times 10^{19} \mathrm{~cm}^{-3}$ ) at $d \geqslant 1 \mathrm{~nm}$.

The profile was fitted by an exponential function as shown by a solid curve in Fig. 2. The same exponential function is also shown by a dotted curve in Fig. 1. This exponential function was convoluted with the resolution function of the spectrometer and the result is shown by a short-dashed curve. The observed energy spectrum was fitted by the sum of three contributions, i.e., the Gauss function representing the surface hydrogen, the convoluted exponential function for the subsurface hydrogen, and the background. The agreement between the fitted result (solid curve) and the observed spectrum is fairly good, suggesting that the present estimation on the subsurface hydrogen profile is reasonable.

Finally, the change of the observed spectrum during the measurement is shown in Fig. 3. The background subtracted spectra are shown together with the fitted results (solid curves: fitted by the sum of the subsurface hydrogen and the surface hydrogen peak). Although the surface hydrogen peak decreases very rapidly due to the ion-stimulated desorption, the subsurface hydrogen tail does not change. This clearly indicates that the observed subsurface hydrogen can be attributed to neither the recoil implantation nor the artificial effects due the surface roughness. If the recoil implantation takes place, we should observe the increase of the subsurface hydrogen tail during the measurement. If the tail is the artificial one due to the surface roughness, the tail should decrease as the surface peak decreases. Thus, the existence of the subsurface hydrogen atoms in the CVD diamond film is confirmed, suggesting that the shallow-acceptor model is likely.

In summary, hydrogen depth profiles are measured for 


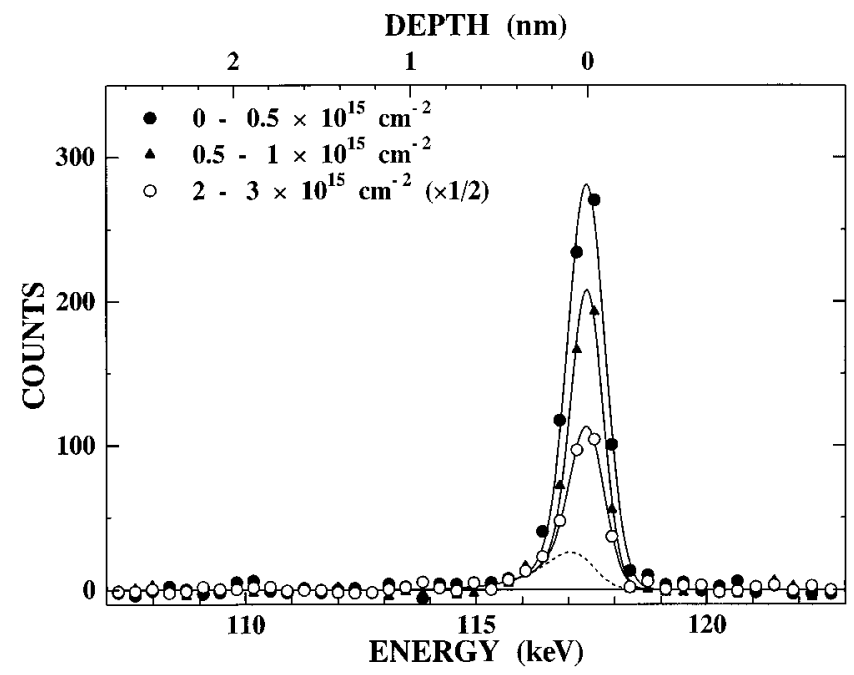

FIG. 3. Change of the energy spectra of recoiled $\mathrm{H}^{+}$during the ERD measurement. The background subtracted spectra observed during the irradiation of $0 \sim 0.5 \times 10^{15} \mathrm{C}^{+}$ions $/ \mathrm{cm}^{-2}$ (closed circle), $0.5 \sim 1 \times 10^{15} \mathrm{C}^{+}$ions $/ \mathrm{cm}^{-2}$ (triangle), and $2 \sim 3 \times 10^{15} \mathrm{C}^{+}$ions $/ \mathrm{cm}^{-2}$ (open circle) are shown. The spectra were fitted by the sum of the subsurface hydrogen (dashed curve) and the surface hydrogen peak as shown by solid curves. The subsurface hydrogen does not change, while the surface hydrogen peak decreases very rapidly due to the ion-stimulated desorption.

CVD diamond films by high-resolution ERD. Most hydrogen atoms are localized at the surface and the surface hydrogen coverage is estimated to be $1.0 \pm 0.3 \mathrm{ML}$, indicating the formation of the monohydride structure. Besides the surface hydrogen, there are some hydrogen atoms incorporated in the subsurface region, which might be the origin of the surface conductivity.

The authors are grateful to the members of the Quantum Science and Engineering Center for use of the 4 MV Van de Graaff accelerator. This work was supported in part by Grant-in-Aid for Scientific Research from the ministry of Education, Science, Sports, and Culture.
${ }^{1}$ H. Watanabe, D. Takeuchi, S. Yamanaka, H. Okushi, K. Kajimura, and T. Sekiguchi, Diamond Relat. Mater. 8, 1272 (1999).

${ }^{2}$ M. I. Landstrass and K. V. Ravi, Appl. Phys. Lett. 55, 975 (1989).

${ }^{3}$ T. Maki, S. Shikama, M. Komori, Y. Sakaguchi, K. Sakuta, and T. Kobayashi, Jpn. J. Appl. Phys., Part 2 31, L1446 (1992).

${ }^{4}$ S. A. Grot, G. Sh. Gildenblat, C. W. Hatfield, C. W. Wronski, A. R. Badzian, T. Badzian, and R. Messier, IEEE Electron Device Lett. 11, 100 (1990).

${ }^{5}$ Y. Mori, A. Hatta, T. Ito, and A. Hiraki, Jpn. J. Appl. Phys., Part 2 31, L1718 (1992).

${ }^{6}$ H. Kiyota, E. Matsushima, K. Sato, H. Okushi, T. Ando, M. Kamo, Y. Sato, and M. Iida, Appl. Phys. Lett. 67, 3596 (1995).

${ }^{7}$ K. Hayashi, S. Yamanaka, H. Okushi, and K. Kajimura, Appl. Phys. Lett. 68, 376 (1996).

${ }^{8}$ K. Hayashi, H. Watanabe, S. Yamanaka, H. Okushi, K. Kajimura, and T. Sekiguchi, Appl. Phys. Lett. 69, 1122 (1996).

${ }^{9}$ H. Kawarada, Surf. Sci. Rep. 26, 205 (1996).

${ }^{10}$ P. Gluche, A. Aleksov, A. Vescan, W. Ebert, and E. Kohn, IEEE Electron Device Lett. 18, 547 (1997).

${ }^{11}$ K. Tsugawa, K. Kitatani, H. Noda, A. Hokazono, K. Hirose, M. Tajima, and H. Kawarada, Diamond Relat. Mater. 8, 927 (1998).

${ }^{12}$ M. I. Landstrass and K. V. Ravi, Appl. Phys. Lett. 55, 1391 (1989).

${ }^{13}$ K. Hayashi, S. Yamanaka, H. Watanabe, T. Sekiguchi, H. Okushi, and K. Kajimura, J. Appl. Phys. 81, 744 (1997).

${ }^{14}$ H. Kawarada, H. Sasaki, and A. Sato, Phys. Rev. B 52, 11351 (1995).

${ }^{15}$ J. Shirafuji and T. Sugino, Diamond Relat. Mater. 5, 706 (1996).

${ }^{16}$ F. Maier, M. Riedel, B. Mantel, J. Ristein, and L. Ley, Phys. Rev. Lett. 85, 3472 (2000).

${ }^{17}$ S. Ri, T. Mizumasa, Y. Akiba, Y. Hirose, T. Kurosu, and M. Iida, Jpn. J. Appl. Phys., Part 1 34, 5550 (1995).

${ }^{18}$ H. Yagi, A. Hara, and T. Ito, Appl. Surf. Sci. 137, 50 (1999).

${ }^{19}$ G. Dollinger, Nucl. Instrum. Methods Phys. Res. B 79, 513 (1993).

${ }^{20}$ K. Kimura, H. Imura, and K. Nakajima, Nucl. Instrum. Methods Phys. Res. B 140, 397 (1998).

${ }^{21}$ J. F. Ziegler, J. P. Biersak, and U. Littmark, The Stopping and Range of Ions in Solids (Pergamon, New York, 1985).

${ }^{22}$ R. S. Gao, P. S. Gibner, J. H. Newman, K. A. Smith, and R. F. Stebbings, Rev. Sci. Instrum. 55, 1756 (1984).

${ }^{23}$ A. Chateu-Thierry and A. Gladieux, in Atomic Collisions in Solids, edited by S. Datz, B. R. Appleton, and C. D. Moak (Plenum, New York, 1975), Vol. 1, p. 307.

${ }^{24}$ H. Tamura, H. Zhou, K. Sugisako, Y. Yoloi, S. Takami, M. Kubo, K. Teraishi, A. Miyamoto, A. Imamura, M. N.-Gamo, and T. Ando, Phys. Rev. B 61, 11025 (2000). 